\title{
Optimization Design of IGV Profile in Centrifugal Compressor
}

\author{
Qi Sun, ${ }^{1,2}$ Chunjun Ji, ${ }^{1,3}$ Junyi Fang, ${ }^{1}$ Chunyang Li, ${ }^{1}$ and Xiaolin Zhang ${ }^{1}$ \\ ${ }^{1}$ School of Energy and Power Engineering, Dalian University of Technology, Dalian 116024, China \\ ${ }^{2}$ Fu-An Gas Turbine Co., Ltd., Liaoning Fu-An Group, Anshan 114044, China \\ ${ }^{3}$ Key Laboratory of Ocean Energy Utilization and Energy Conservation of Ministry of Education, Dalian University of Technology, \\ Dalian 116024, China \\ Correspondence should be addressed to Qi Sun; sunqi525@126.com
}

Received 23 September 2017; Revised 7 November 2017; Accepted 21 November 2017; Published 19 December 2017

Academic Editor: Benjamin Ivorra

Copyright (C) 2017 Qi Sun et al. This is an open access article distributed under the Creative Commons Attribution License, which permits unrestricted use, distribution, and reproduction in any medium, provided the original work is properly cited.

\begin{abstract}
Variable inlet guide vane (IGV) is used to control the mass flow and generate prewhirl in centrifugal compressors. The efficient operation of IGV is limited to the range of aerodynamic characteristics of their vane profiles. In order to find out the best vane profile for IGV regulation, the modern optimization method was adopted to optimize the inlet guide vane profile. The main methodology idea was to use artificial neural network for continuous fitness evaluation and use genetic algorithm for global optimization. After optimization, the regulating performance of IGV has improved significantly, the prewhirl ability has been enhanced greatly, and the pressure loss has been reduced. The mass flow and power of compressor reduced by using the optimized guide vane at large setting angles, and the efficiency increased significantly; the flow field distribution has been improved obviously, since the nonuniform distribution of flow and flow separation phenomenon greatly weakened or even completely disappeared. The achievement of this research can effectively improve the regulation ability of IGV and the performance of compressor.
\end{abstract}

\section{Introduction}

In order to adapt to climate change and to meet the user requirements, the gas mass flow in actual operation of a centrifugal compressor needs to be regulated normally [1]. Variable inlet guide vane (IGV) in the first stage of a centrifugal compressor is one of the effective ways to realize this function [2]. However, the regulating ability of traditional IGV is very limited, resulting in a large amount of compressed gas that has to be discharged without effective use because of overproduction, especially in winter, up to $30 \%$ in some cases [3]. So it is very important to develop high efficiency IGV device to solve the problem.

IGV is installed in the inlet of compressor to regulate mass flow (Figure 1). Most IGVs have symmetric vane profiles and create similar inlet flow characteristics at positive and negative prewhirls. At nonzero setting angles, flow experiences strong suction side acceleration in the vicinity of the leading edge. The subsequent large deceleration can cause massive flow separation already at low incidence, which limits the useful operating range of IGV [4].
Usually, researchers pay more attention to the impeller rather than the inlet guide vane, since it is a stationary part in centrifugal compressor. Therefore, there are few designers using modern optimization methods to do optimization design for inlet guide vane profile. In practical application, the inlet guide vanes are used with the existing NACA profile. Cao et al. [5] had put forward the design of the three-dimensional space of blade in turbomachinery, which could provide reference for the design of IGV in centrifugal compressor. Zhang et al. [6-9] had tested a straight IGV in centrifugal fan and analyzed the flow characteristics in IGV channel. It was found that the flow parameters of gas tend to decrease along with the flow direction. According to the distribution characteristics of airflow in the IGV channel, the profile of IGV should be improved. After repeated improvements and experiments, an asymmetric vane named B20L45RF has been developed to improve the regulating performance of IGV in centrifugal fan. Swain [10] has carried out CFD tests on flat straight vanes ranging from $20^{\circ}$ to $90^{\circ}$; the results show that when the guide vane setting angle is large (about $60^{\circ}$ ), the flow separation is produced on the suction 


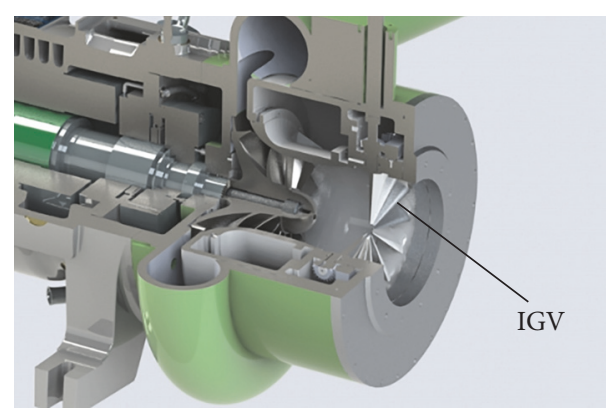

FIGURE 1: IGV in centrifugal compressor.

side of the guide vane, and the development of the separation deteriorates the flow of the guide vane channel and makes the total loss of the system increase. To solve this problem, Coppinger and Swain [11] proposed combinatorial tandem vane. Tandem vane ensures that the airflow direction into the guide vane passage is consistent with the leading edge of the guide vane. The front part of guide vane is stationary; the back part of guide vane can be turned by rotating the shaft to achieve the flow regulation. Thus, minimum angle of attack can be reached at any setting angle, the flow separation is weakened, and the aerodynamic performance of the system is improved. But the structure of tandem vane regulating device is complex and the cost is very expensive. And this kind of guide vane also has other problems, such as the increase of guide vane chord; the back part of the guide vane bears a greater torque. Subsequently, Mohseni et al. $[2,12]$ investigated the thickness and camber of guide vane profile and designed a nonsymmetry (S-Cambered) guide vane by converse proposition design method. To compare with the tandem vane, the S-Cambered vane shows better performance.

At present, the genetic algorithm is more commonly used for the optimization design of blade in turbomachinery. However, the evolution process of the genetic algorithm needs a lot of fitness and needs a large number of invoking flow calculation procedures, so that the convergence speed is very slow. The optimization of this paper is based on the approximate function technology. The aerodynamic performance of guide vane can be rapidly evaluated by approximate function model. This evaluation process can simulate the real performance of guide vane as fast as possible and without the time-consuming calculation of flow field. According to the samples generated by the database, the approximate function relationship between the geometric optimization variables and the parameters of the aerodynamic target is established by using artificial neural network. On this basis, the genetic algorithm is used to optimize and predict the optimal solution. Then, the predicted results are checked by $\mathrm{N}-\mathrm{S}$ flow field numerical calculation, and the results are added to the database sample to generate new approximate functions. The optimal solution satisfying different optimization objectives is searched repeatedly. The optimization of guide vane combining genetic algorithm and artificial neural network is adopted in this paper, eliminating a large number of flow field calculations, which can greatly reduce the optimization time.

\section{The Evaluation of IGV Regulation Performance}

The ideal IGV regulation system should be able to produce larger prewhirl while minimizing its own losses. We have summarized two evaluation criteria of the performance of IGV regulation, prewhirl coefficient and total pressure loss coefficient.

2.1. Prewhirl Coefficient. When the centrifugal compressor adopts inlet guide vanes to regulate, the prewhirl is generated by changing the intake direction, thereby changing the working capacity of the impeller to the gas and realizing the regulation of variable working condition of compressor. Therefore, the ability of the vane to generate prewhirl is an important index to evaluate its performance.

If the airflow gets into the impeller after IGV regulation without loss, according to the principle of momentum conservation, the movement of the airflow in the region will keep momentum unchanged. The cross section at the exit of the guide vane is taken as the object of study. First, we assume the airflow is incompressible before entering into impeller, so the density of gas is constant. We assume the prewhirl angle generated by inlet guide vane is consistent with the setting angle of guide vane, ignoring its deviation and assume the distribution of axial velocity $V_{z}$ and circumferential velocity $V_{\theta}$ is uniform at the exit section of guide vane channel. Then, the momentum torque $M_{z}$ obtained after the regulation of guide vane is expressed as follows:

$$
\begin{aligned}
M_{z} & =\int_{S} r V_{\theta} d m=\int_{R_{H}}^{R_{S}} r V_{\theta}\left(2 \pi r V_{z} \rho d r\right) \\
& =\overline{V_{\theta}} \cdot \bar{V}_{z} \rho \int_{R_{H}}^{R_{S}} 2 \pi r^{2} d r=\overline{V_{\theta}} \cdot \pi \bar{V}_{z} \rho \cdot \frac{2\left(R_{S}^{3}-R_{H}^{3}\right)}{3} .
\end{aligned}
$$

$R_{H}$ and $R_{S}$ are the inner radius and the outer radius of the exit section of IGV channel. $\bar{V}_{\theta}$ and $\bar{V}_{z}$ are the mass averaged values of the circumferential velocity $V_{\theta}$ and axial velocity $V_{z}$ at this cross section. $m$ is mass flow, which can be expressed as

$$
m=A_{1} \bar{V}_{z} \rho=\pi\left(R_{S}^{2}-R_{H}^{2}\right) \bar{V}_{z} \rho .
$$

The equivalent prewhirl radius $R_{1}$ of this section is defined as

$$
R_{1}=\frac{2\left(R_{S}^{3}-R_{H}^{3}\right)}{3\left(R_{S}^{2}-R_{H}^{2}\right)}
$$

The numerator and denominator of formula (1) are multiplied $\left(R_{S}^{2}-R_{H}^{2}\right)$ at the same time:

$$
M_{z}=\overline{V_{\theta}} \cdot \pi\left(R_{S}^{2}-R_{H}^{2}\right) \bar{V}_{z} \rho \cdot \frac{2\left(R_{S}^{3}-R_{H}^{3}\right)}{3\left(R_{S}^{2}-R_{H}^{2}\right)}=\overline{V_{\theta}} m R_{1} .
$$


In order to quantitatively describe the prewhirl effect of IGV regulation, introduce the concept of prewhirl coefficient, which is defined as

$$
\lambda=\frac{M_{z}}{m \bar{V}_{z} R_{1}}=\frac{\overline{V_{\theta}}}{\bar{V}_{z}} .
$$

The magnitude of prewhirl coefficient represents the prewhirl capacity of IGV regulation. The purpose of installing IGV in centrifugal compressors is to produce sufficient prewhirl to achieve the purpose of flow regulation. Therefore, the greater the prewhirl coefficient value of IGV at a certain setting angle, the better.

2.2. Pressure Loss Coefficient. The flow loss or flow efficiency is regarded as one of the evaluation criteria for evaluating the performance of IGV, as in other static flow components of fluid machinery. In this paper, the total pressure loss coefficient $\xi$ is used to quantitatively evaluate the flow loss of IGV. It is defined by the ratio of the change in mass averaged stagnation pressure between the IGV inlet and exit and the average dynamic pressure at the IGV inlet:

$$
\xi=\frac{\bar{p}_{t 0}-\bar{p}_{t 1}}{\bar{p}_{t 0}-\bar{p}_{s 0}}=\frac{\Delta \bar{p}_{t-\mathrm{VIGV}}}{(1 / 2) \rho_{0} \bar{V}_{0 z}^{2}} .
$$

$\bar{p}_{t 0}$ and $\bar{p}_{t 1}$ represent the mass average of the total pressure at the inlet section and exit section of the IGV channel, respectively; $\bar{p}_{s 0}$ is the mass average of the static pressure at the inlet section; $\Delta \bar{p}_{t \text {-VIGV }}$ is the total pressure loss of airflow through the IGV passage. $\rho_{0}$ is the density of gas at the inlet section. $\bar{V}_{0 z}$ is the average of axial velocity at the inlet section.

The pressure loss coefficient represents the magnitude of the flow loss in the IGV channel, so the value of the pressure loss coefficient should be reduced as much as possible.

\section{Optimization of IGV Profile}

3.1. Parametric Fitting of Guide Vane Profile. The parameterization is to describe the guide vane profile with finite design parameters so as to control and modify the guide vane profile. The number of design parameters determines the variability of guide vane profile and also affects the amount of design work. The more the design parameters, the greater the design workload. Therefore, it is very important to select the minimal design variables to express the profile of the guide vane in the process of optimization.

Currently, $B$-spline curve and Bezier curve are commonly used for parameterization of blade profile. Because $B$-spline curve interpolation function compared to the Bezier curve is more complex, it is suitable for capturing the local shape of blade, and it has more control points and produces more design variables, not suitable for global optimization of guide vane. Bezier curve is more suitable for describing the profile of blade with the properties of convexity and smoothness, and the construction of function is relatively simple; change in any control point can change the shape of the entire curve; it only needs less control points. The optimization of the guide

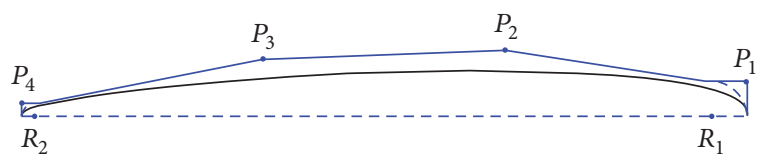

FIGURE 2: Curve fitting of guide vane.

vane is global, so Bezier curve is determined to be used as the parametric fitting of guide vane.

The optimization design that was done in this paper is based on the original IGV profile. In order to achieve a good fitting effect and minimize the error between the original data and fitting data, the least squares method was used. In order to ensure the aerodynamic configuration requirements of IGV, the Bezier curve [13-15] based on Bernstein basis function was used to describe the IGV profile.

The vane profile is similar to the airfoil and its special geometrical characteristics; the suction and pressure sides were separately fitted, so there are two curves in each section [16]. Bezier curves with different order (three orders, five orders, seven orders, and ten orders) were used to conduct parametric fitting for the airfoil. The results show that the profile of airfoil can be fully described by seven-order and ten-order Bezier curve, while the three-order and five-order Bezier curves show obvious sharp corners at the leading edge and trailing edge of the airfoil [17]. Since the endpoints of the two curves are coincident $[18,19]$, but the first-order derivative of these two endpoints is not continuous, there will be obvious sharp corners. The endpoints should be treated specially. In order to solve this problem, the leading edge and the trailing edge are expressed in the form of radius. The radii of leading edge and trailing edge and the Bezier control points are used together as the design variables to adjust the vane profile. Although the leading edge and trailing edge of guide vane are simplified into arcs without more careful optimization, it can avoid the formation of sharp corners at endpoints and keep the aerodynamic requirement of vane profile. Finally, in the premise of ensuring the fitting precision, in order to minimize the number of control variables, three-order Bezier curves combined with special treatment on the endpoints were used to conduct parametric fitting for the guide vane profile and achieved good fitting effect.

Figure 2 shows the fitting result of guide vane suction side, the distance between $P_{1}$ and the leading edge equal to the leading edge radius $R_{1}$, and the distance between $P_{4}$ and the trailing edge equal to the trailing edge radius $R_{2}$, so the guide vane suction side can be described by the four design variables $P_{1}, P_{2}, P_{3}, P_{4}$.

3.2. Objective Function. Objective function is the key factor in the process of optimization. The ideal IGV regulation system should be able to produce as much as possible the prewhirl, and its own losses should be as small as possible. In order to enhance the prewhirl ability of guide vane, the prewhirl coefficient is taken as one of the optimization objectives. In addition, in order to reduce the flow losses, the pressure loss coefficient is taken as another optimization 
objective. Therefore, this optimization has two optimization objectives, and the two optimization objectives will be mutually restricted. If the multiobjective optimization is used, the obtained solution is usually a set of optimal solutions, and it is difficult to find a global optimal solution. Therefore, it is necessary to transform multiobjective optimization into single objective optimization. According to the optimization objective of this research, we need to obtain the maximum value of the prewhirl coefficient and the minimum value of the pressure loss coefficient. In order to combine the two parameters into a single objective function, we put the pressure loss coefficient at the denominator. Thus, the optimization of the guide vane profile is to maximize $\delta=$ $\lambda / \xi$ as the objective function. The design variables are the Bezier curve control points on the guide vane profile and the leading and trailing angle. The geometric constraint conditions are the vane thickness, the setting angle, the leading edge angle, and the trailing edge angle of guide vane. Finally, the expression of the optimization objective function is

$$
\begin{array}{ll}
\max & \delta\left(P_{n m}^{S}, P_{n m}^{P}, \beta_{1 m}, \beta_{2 m}\right)=\frac{\lambda}{\xi}=\frac{\rho_{0} \bar{V}_{0 z}^{2} \bar{V}_{\theta}}{2 \Delta \bar{p}_{t-\mathrm{VIGV}} \bar{V}_{z}} \\
\text { s.t. } & 0.6 D_{0} \leq D \leq 1.6 D_{0}, \\
& \gamma \equiv 60^{\circ} \\
& 0^{\circ} \leq \beta_{1 m} \leq 60^{\circ} \\
& 0^{\circ} \leq \beta_{2 m} \leq 60^{\circ} \\
& \lambda \geq \lambda_{0} \\
& \xi \leq \xi_{0},
\end{array}
$$

where $\lambda$ is prewhirl coefficient and $\xi$ is total pressure loss coefficient. $P_{n m}^{S}$ and $P_{n m}^{P}$ represent the design variables control points of suction side and pressure side, respectively. $\beta_{1 m}$ and $\beta_{2 m}$ represent the leading edge angle and the trailing edge angle, respectively, where the numbers $n=1,2,3,4$ represent the four control points on the Bezier fitting curve, and the $m=1,2,3$ represent the three sections (bottom, middle, and top of guide vane). Then, $n$ and $m$ are selected sequentially, 30 design variables can be obtained. $D$ represents the thickness of the optimized guide vane, and $D_{0}$ represents the thickness of the original guide vane. The thickness of the optimized guide vane is not less than 0.6 times and no more than 1.6 times of the original one. $\gamma$ represents the setting angle of the guide vane, which is limited to $60^{\circ}$. The leading edge and the trailing edge angle of the guide vane vary from $0^{\circ}$ to $60^{\circ}$, respectively. $\lambda_{0}$ and $\xi_{0}$ are the original guide vane's prewhirl and pressure loss coefficient. In the optimization process, the prewhirl coefficient is not less than the original guide vane; the pressure loss coefficient is not higher than that of the original one.

The objective function and constraints selected in this paper are based on our previous research results on IGV profile [3]. The objective function can fully describe the performance of IGV itself, and the constraints are also enough to meet the requirements, since the optimization result is reasonable. In order to avoid convex problem with global optimum, single objective function was adopted to optimize the inlet guide vane profile. Artificial neural network was used for continuous fitness evaluation and genetic algorithm for global optimization. The whole computational time can be reduced within 72 hours. In the process of optimization, the evolution of the design variables is calculated by calling the CFD program and compared with the initial design variables until the optimal solution is obtained.

3.3. Optimization Steps. The process of optimization design of IGV is shown in Figure 3. Before the optimization of IGV was carried out, the design variables, objective functions, and constraints should be determined first. Then, the parametric fitting was carried out based on Bezier curve; the purpose is to use fewer design variables to control hundreds of point of the original vane profile, so it can greatly reduce the number of design variables. The third step of the optimization was to generate the sample database based on the principle of uniform design. The design variables are 30, and 200 database samples were generated. The fourth step was to analyze the performance of each different guide vane profiles in the sample database by using CFD technology and calculated the optimal parameters of the guide vanes of each sample point. The fifth step was to train the sample database by using artificial neural network technology [20] and established the sample database of guide vane optimal variables and the performance evaluation of approximate model parameters and then calculated the performance parameters of different vane profiles in nonsample database. The sixth step was to use genetic algorithm [21] for global optimization, to find making the target function reach the best profile of guide vane in the approximate model with the constraints and to take it as the current optimal prediction vane profile. The seventh step was to use the CFD calculation again to analyze the performance of the current optimal prediction vane profile. The calculated objective function value was compared with those of the current optimal prediction vane profile. If the objective function prediction of vane profile was better than the current optimal objective function value, it would replace the current optimal vane profile. Otherwise, it was added to the sample database as a sample point to continue training and returned to the fifth step to generate new approximate model using artificial neural network technology. The last step was to judge the convergence condition of the optimization. If the convergence condition was satisfied, the optimization was terminated and the optimal vane profile was obtained. If the convergence condition was not satisfied, a new iteration was started until the convergence condition was satisfied. The convergence criterion of the optimization was that the objective function value was four consecutive steps in the optimization process, and the increment of the numerical value was less than $1 \%$.

\section{Results and Discussion}

Through the above optimization process, the bottom, the middle, and the top of the vane section profiles were 


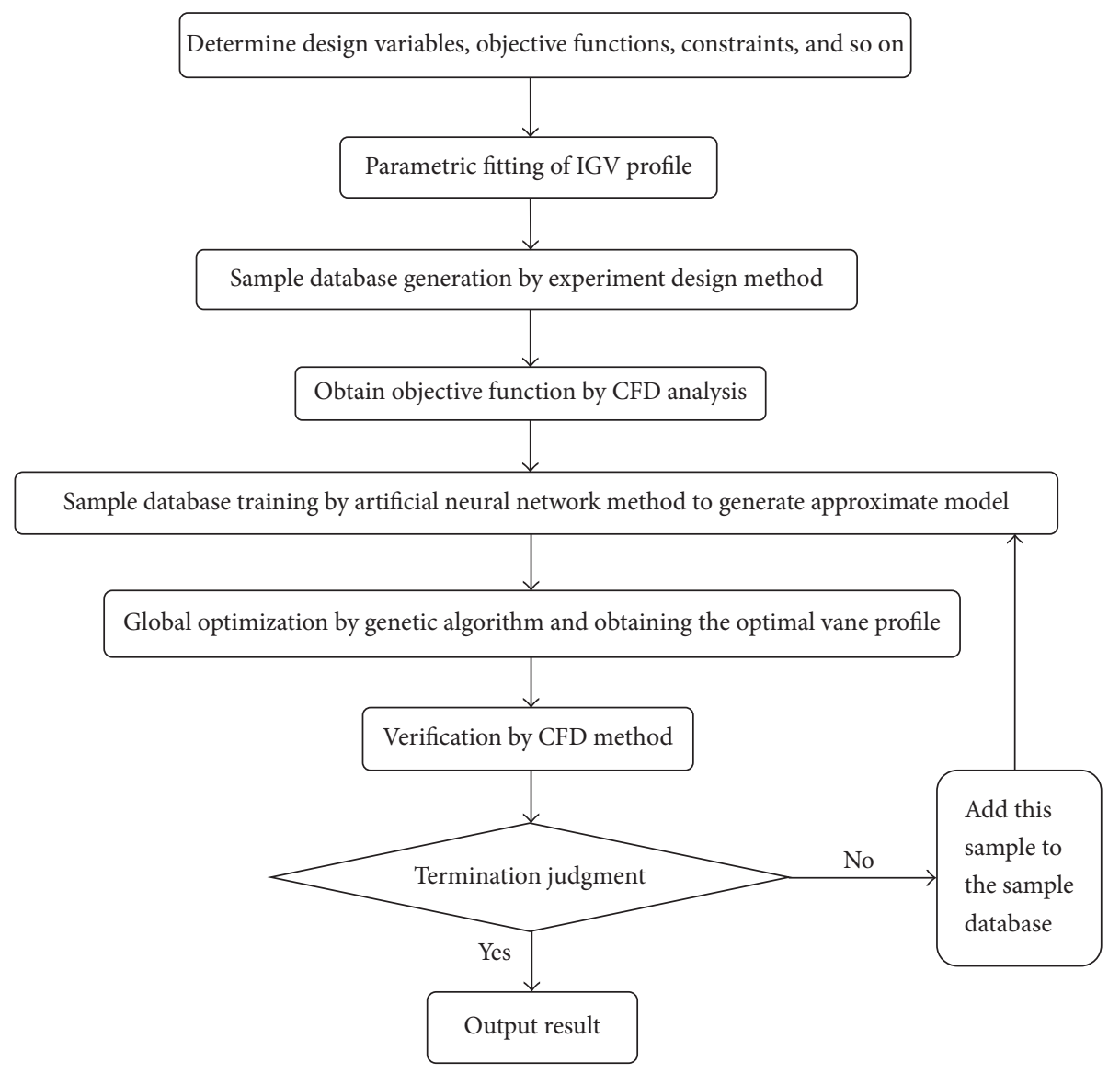

FIgURE 3: Flow chart of inlet guide vane optimization.

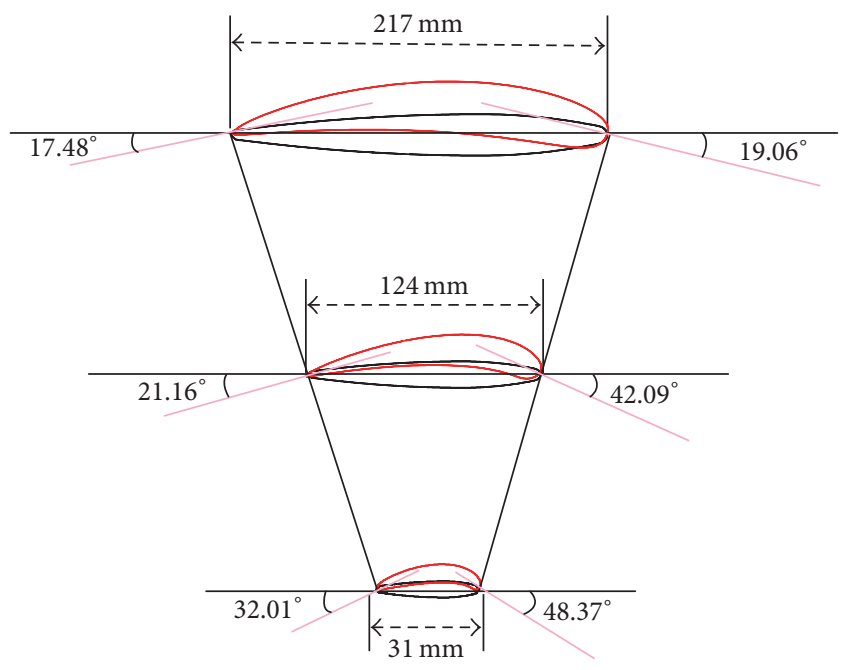

- Original

— Optimized

FIGURE 4: Comparison of the original and the optimized profile.

optimized and compared with the original one, as shown in Figure 4. By comparison, we found that the thickness of optimized vane increased compared with the prototype, and the thickness is bigger along with the vane from bottom to top, especially near the leading edge, more conducive to adapt to the flow of gas. From the bottom to the top, the vane camber decreased significantly. Because of the worst flow of gas at the bottom of vane, flow separation is prone to occur. By increasing the curvature at the bottom of the vane, the flow separation phenomenon is reduced and the flow loss is reduced. In addition, the maximum deflection position of the three sections is in the first half of the guide vane, and closer to the leading edge from top to bottom.

After optimization, the pressure loss coefficient of the guide vane is reduced by 1.02027 , and the prewhirl coefficient is increased by 0.66518 , which significantly improves the prewhirl capability of the guide vanes and greatly reduces the pressure loss. For compressor performance, the flow rate is reduced by $17.67 \%$, the power is reduced by $18 \%$, the pressure ratio is reduced by only 0.007 , and the efficiency is increased by $0.94 \%$, shown in Table 1 .

The optimization was carried out at $60^{\circ}$ setting angle of guide vane. In order to explore the performance of optimized guide vane at other setting angles, the performance parameters had been tested at $-20^{\circ}, 0^{\circ}, 20^{\circ}, 40^{\circ}$, and $60^{\circ}$ and compared with the original ones, as shown in Figure 5. 


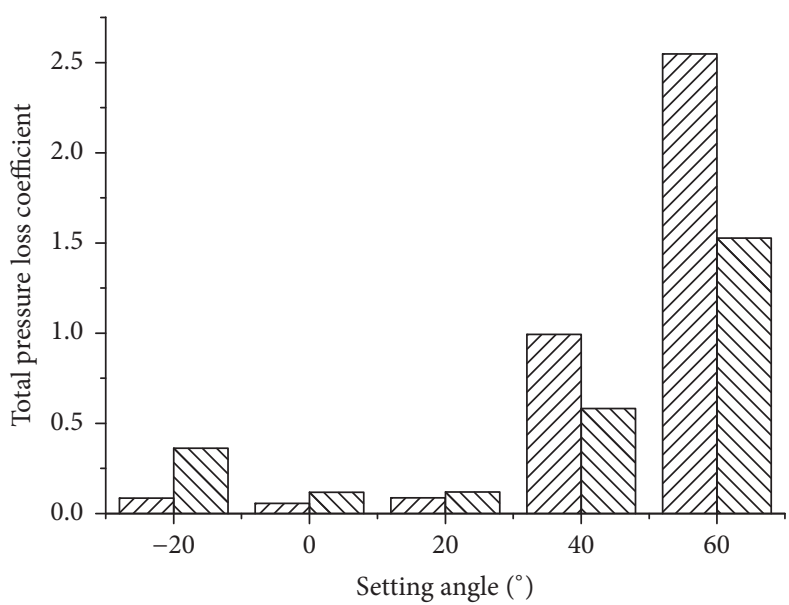

WII Original profile IN Optimized profile

(a) Pressure loss coefficient

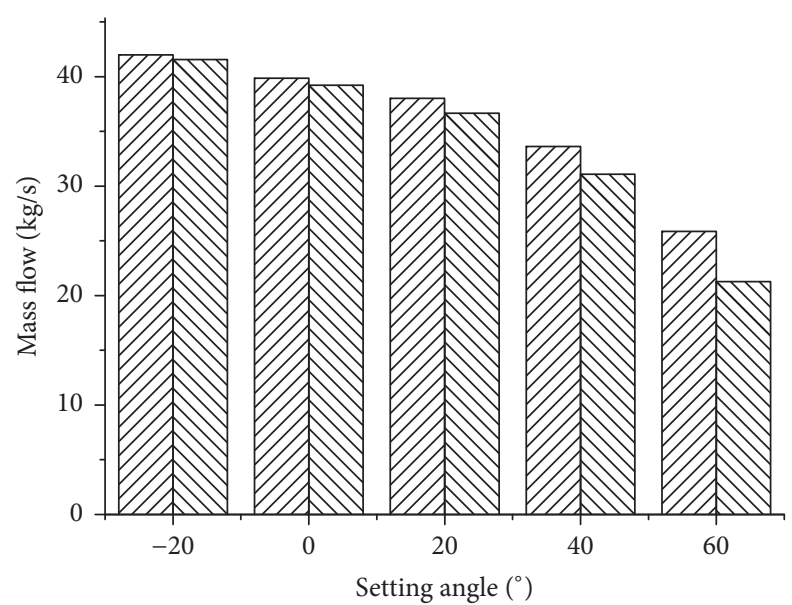

VIIA Original profile

Optimized profile

(c) Mass flow

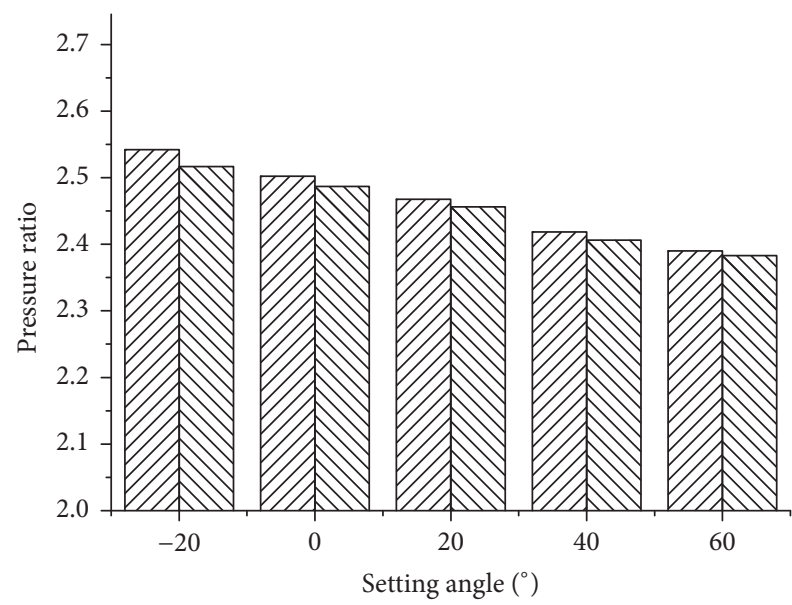

VIIt Original profile MV Optimized profile

(e) Pressure ratio

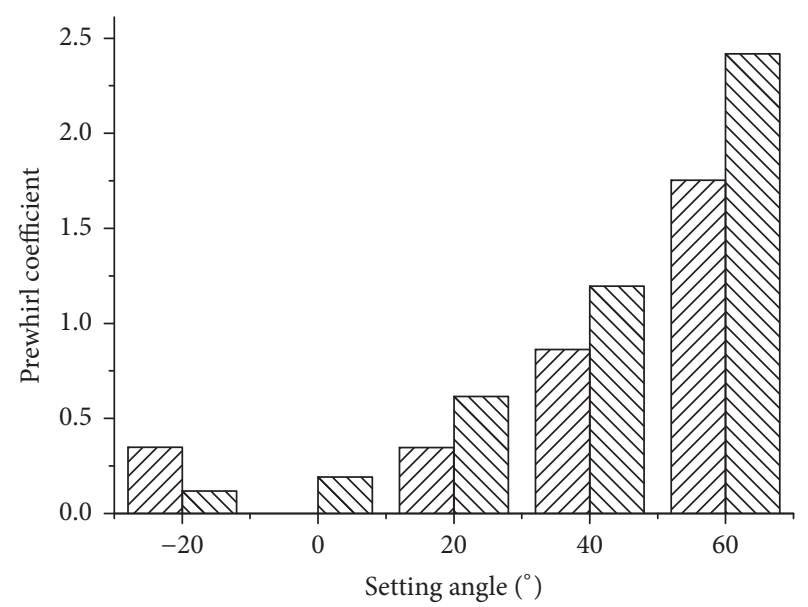

VIIS Original profile

MVtimized profile

(b) Prewhirl coefficient

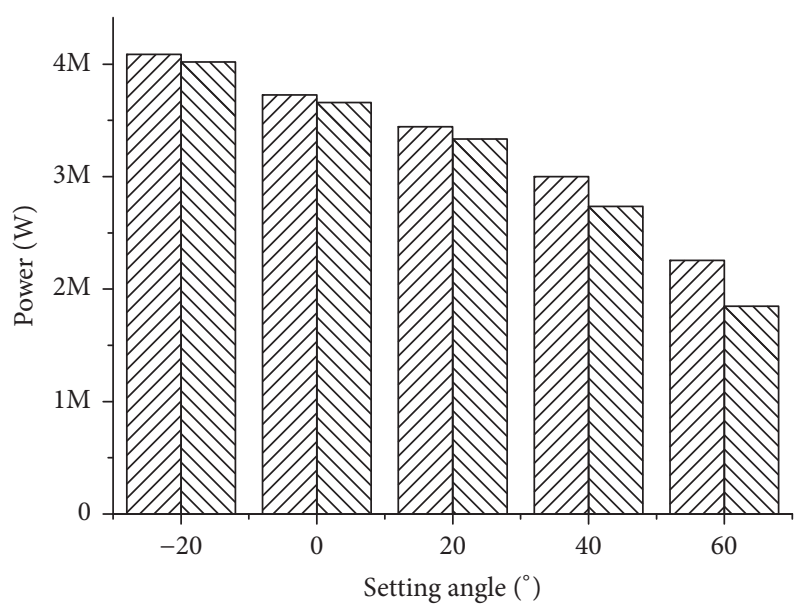

VIIA Original profile

Optimized profile

(d) Power

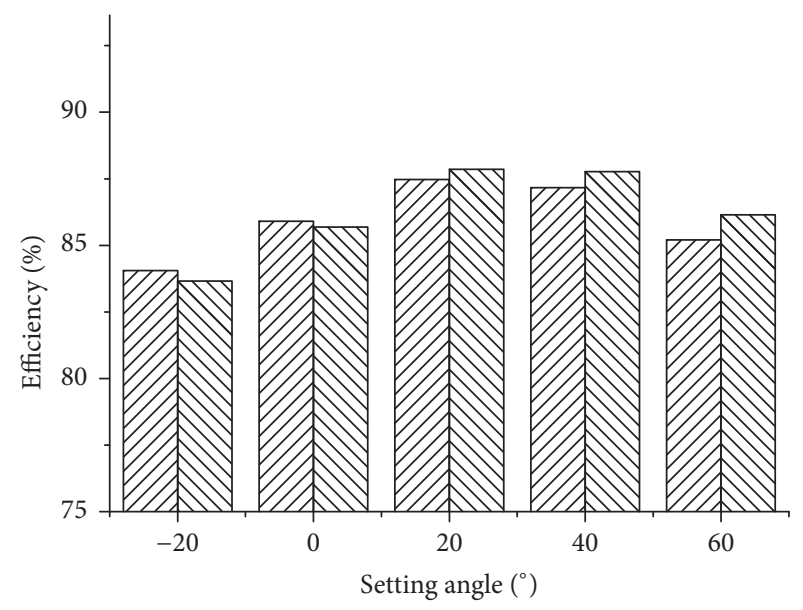

VIIA Original profile MVtimized profile

(f) Efficiency

FIGURE 5: The comparison of the performance parameters of the guide vane and the compressor at each setting angle. 


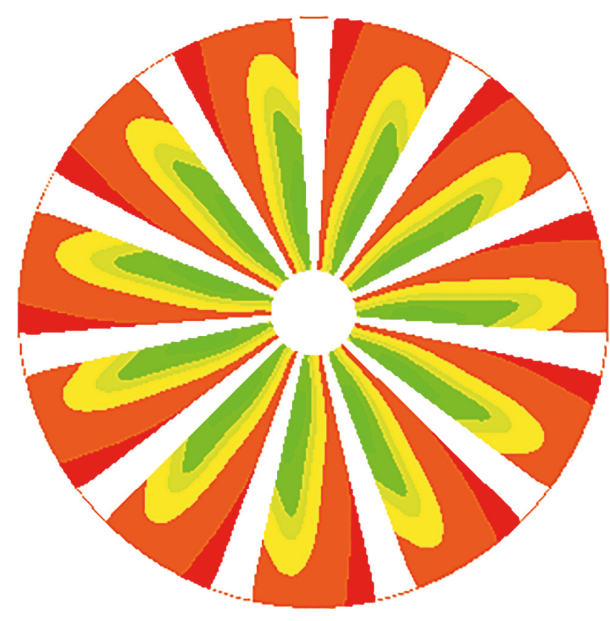

(a) Original profile
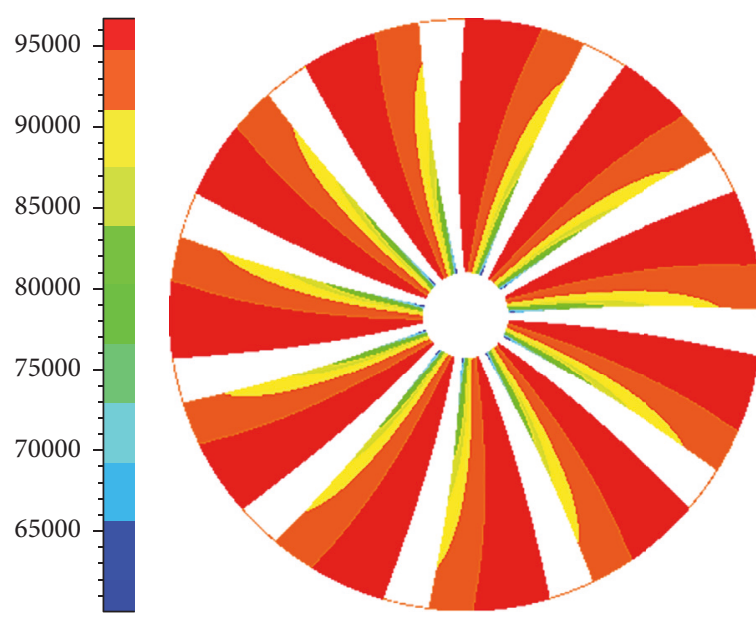

(b) Optimized profile

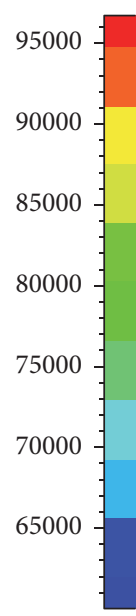

FIGURE 6: Comparison of static pressure distribution in the flow passage of the optimized guide vane and the original one.

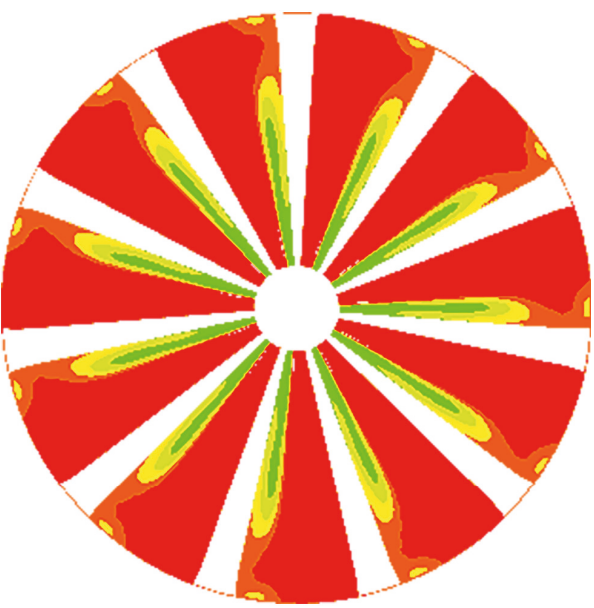

(a) Original profile
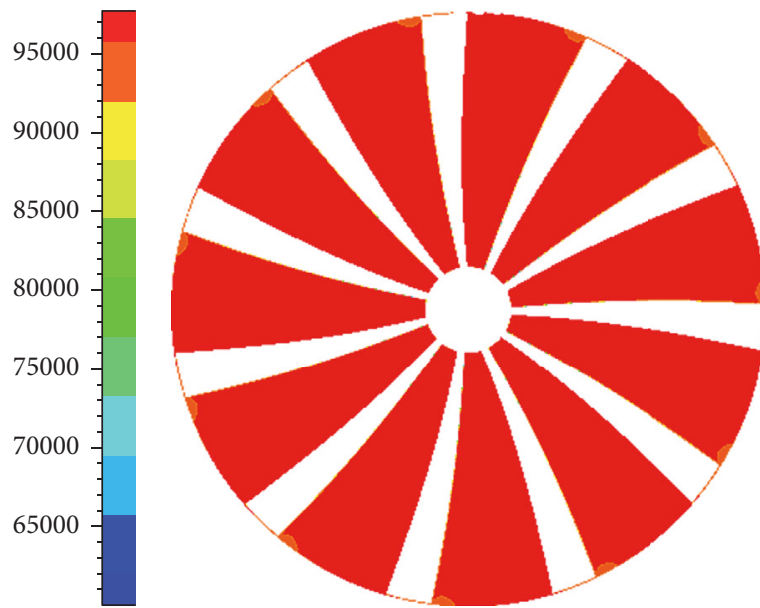

(b) Optimized profile

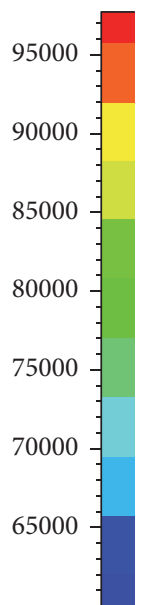

FIGURE 7: Comparison of absolute total pressure distribution in the flow passage of the optimized guide vane and the original one.
The optimized guide vane profile can greatly reduce the pressure loss of the guide vane at large setting angles and has little effect on that at small setting angles, while the pressure loss increased slightly at $-20^{\circ}$ setting angle. When the guide vane is in positive prewhirl regulation, the optimized vane profile can effectively improve the prewhirl capability of the guide vane, and the greater the setting angle of the guide vane, the greater the magnitude of the increase. When the guide vane is in negative prewhirl regulation, the prewhirl capability of the optimized guide vane is slightly decreased.

By adopting the optimized guide vanes, the mass flow and power of the compressor can be reduced at each setting angle and from $-20^{\circ}$ to $60^{\circ}$; with the increase of the setting angle of the guide vane, the magnitude of the reduction is greater. The optimized guide vane reduces the pressure ratio of the compressor slightly, especially at $-20^{\circ}$ setting angle. The optimized vane can improve the efficiency of the compressor at positive prewhirl regulation, and the greater the setting angle is, the more obvious the efficiency increases, but there is a small decrease at $0^{\circ}$ and $-20^{\circ}$.

Therefore, the optimized guide vane greatly improves the regulating ability of the guide vane and the performance of compressor when it is in positive prewhirl regulation, but there are some disadvantages in negative prewhirl regulation. Since the setting angle of guide vane is limited in the negative prewhirl regulation, the disadvantages will not expand. Overall, the optimized vane profile improves the performance of guide vane regulation.

Figures 6 and 7 show the comparison of static pressure and absolute total pressure distribution in the flow passage of the optimized guide vane and the original one. The optimized guide vane profile obviously reduces the static pressure 


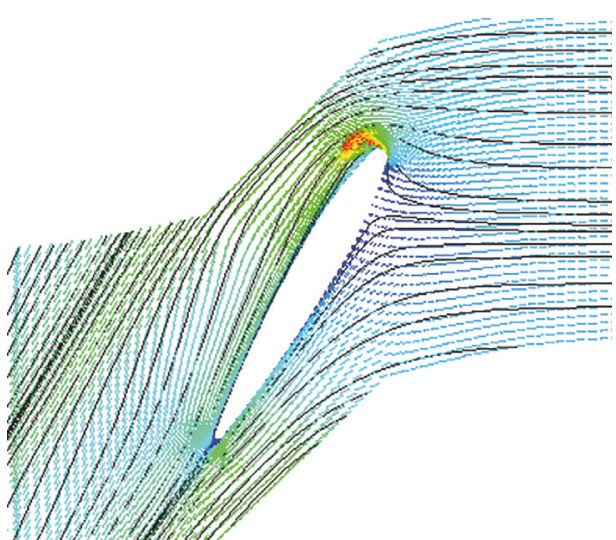

$90 \%$

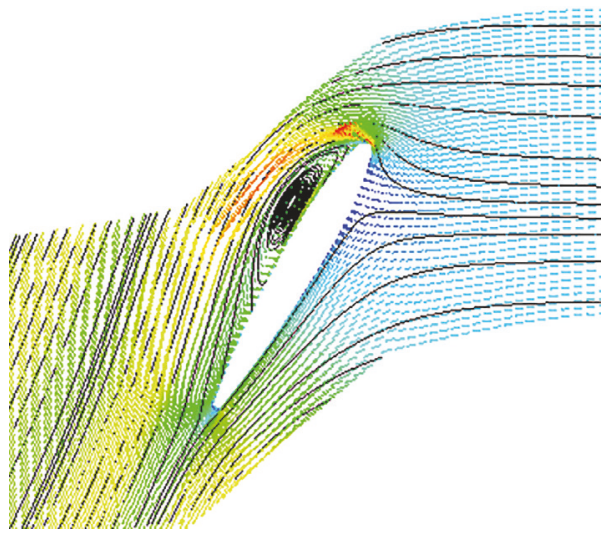

$50 \%$

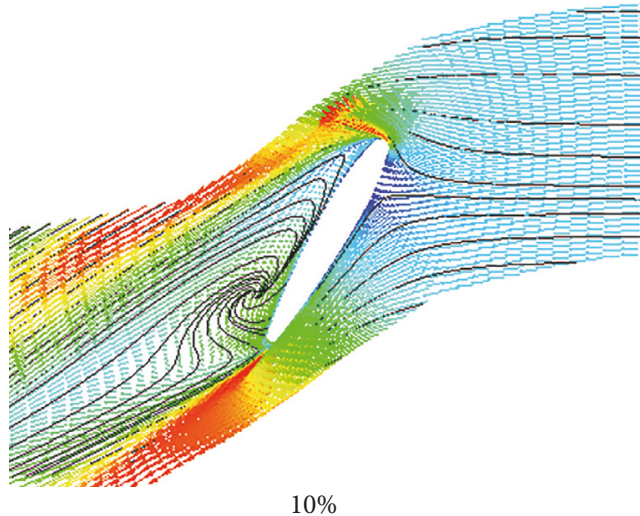

(a) Original profile

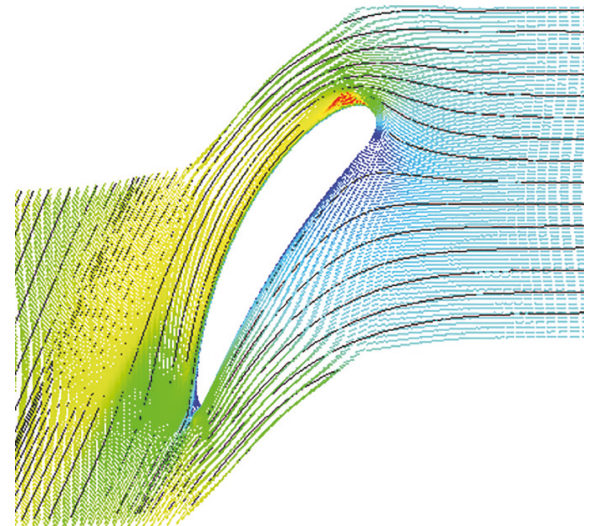

$90 \%$

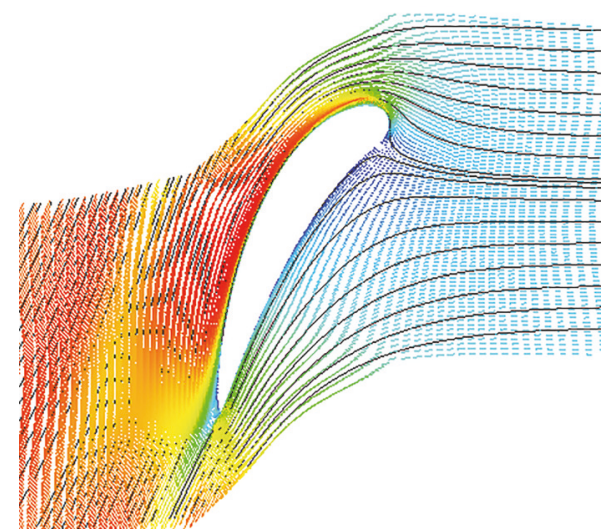

$50 \%$

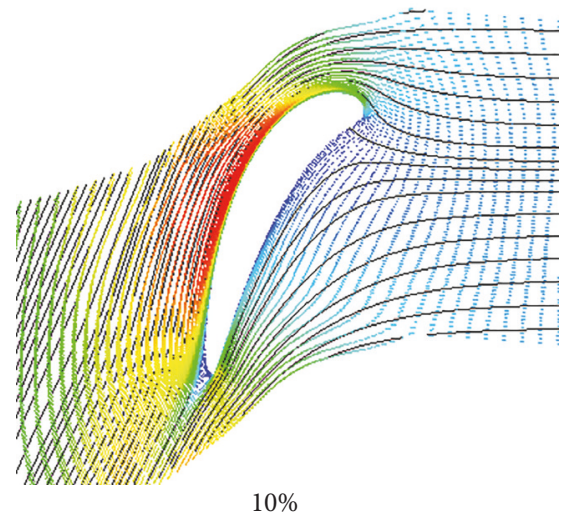

(b) Optimized profile

FIGURE 8: Comparison of velocity vector and streamline of the optimized guide vane's blade to blade section and the original one.

TABLE 1: Parameters of guide vane regulation before and after optimization.

\begin{tabular}{lcc}
\hline performance parameter & Original profile & Optimized profile \\
\hline Pressure loss coefficient & 2.54793 & 1.52766 \\
Prewhirl coefficient & 1.75281 & 2.41799 \\
Mass flow (kg/s) & 25.86 & 21.29 \\
Power (MW) & 2.2549 & 1.8479 \\
Pressure ratio & 2.39 & 2.383 \\
Efficiency (\%) & 85.21 & 86.15 \\
\hline
\end{tabular}

gradient of the suction side of the guide vane and makes the low pressure area of total pressure of suction side disappear completely; the pressure distribution is more uniform.

Figure 8 shows the comparison of velocity vector and streamline of the optimized guide vane's blade to blade section with the original one. Large vortex appears at the suction side of the $50 \%$ and $10 \%$ cross section of the original guide vane; the flow losses are very serious. The optimized guide vane completely eliminates the vortex of the suction side, and the flow of gas is smoother. 


\section{Conclusion}

In this paper, the prewhirl coefficient and the total pressure loss coefficient are used as the evaluation indexes for the performance of IGV regulation. The artificial neural network and genetic algorithm are combined to optimize the vane profile of IGV. The ideal IGV profile has been obtained after optimization, and the regulating performance of the guide vane is significantly improved. The optimized guide vane increases its prewhirl capability and reduces its pressure loss greatly. The optimized vane profile makes the pressure field distribution more uniform in the guide vane channel and the vortex of the suction side of the guide vane has completely disappeared, and the flow field distribution in the whole channel has been greatly improved. The optimized IGV can help to reduce the mass flow and the power of compressor and increase the efficiency. Therefore, the optimized profile can greatly improve the regulating performance of IGV.

\section{Conflicts of Interest}

The authors declare that there are no conflicts of interest regarding the publication of this paper.

\section{References}

[1] J. Tan, Y. Mao, D. Qi, R. Wang, and X. Wang, "Research overview on adjustable inlet guide vane for centrifugal compressor," Compressor, Blower \& Fan Technology, vol. 3, pp. 44-48, 2006.

[2] A. Mohseni, E. Goldhahn, R. A. Van den Braembussche, and J. R. Seume, "Novel IGV designs for centrifugal compressors and their interaction with the impeller," Journal of Turbomachinery, vol. 134, no. 2, Article ID 021006, 8 pages, 2012.

[3] C.-J. Ji, Z.-Y. Fan, Y. Zhang, and W.-H. Ji, "Study on blade profiles of variable inlet guide vanes of centrifugal compressor," Journal of Thermal Science and Technology, vol. 12, no. 2, pp. 131134, 2013.

[4] Y. N. Chen, D. Hagelstein, I. Kassens, H. Hasemann, U. Haupt, and M. Rautenberg, "Overshoot of the rankine vortex formed in the flow field behind the inlet guide vane of centrifugal compressors," in Proceedings of the ASME International Gas Turbine and Aeroengine Congress and Exhibition, Paper no. 99GT-182, Indianapolis, Ind, USA, June 1999.

[5] S. Cao, L. Tan, and S. Gui, "Design and test of front guide vane of centrifugal pump," Transactions of the Chinese Society for Agricultural Machinery, vol. 41, no. S1, pp. 1-5, 2008.

[6] Y. Zhang, D. Qi, and Y. Mao, "Experimental investigation and improvement of the inlet guide vane with plate vane in a centrifugal fan," Proceedings of the Institution of Mechanical Engineers, Part A: Journal of Power and Energy, vol. 223, no. 4, pp. 401-413, 2009.

[7] Y. Zhang, Research on Adjusting Characteristics of Inlet Guide Vanes and Performance Prediction of Centrifugal Fan, Jiao Tong University, Xi’an, China, 2011.

[8] Y. Zhang, D. Qi, and J. Tan, "Experimental research on improving gate performance based on blade shape and blade solidity," Academic Journal of Xian Jiaotong University, vol. 42, no. 5, pp. 583-587, 2008.

[9] Y. Zhang, D. Qi, and J. Tan, "Experimental study on regulating gate of straight blade of centrifugal fan," Academic Journal of Xian Jiaotong University, vol. 41, no. 9, pp. 1044-1048, 2007.
[10] E. Swain, "The design of an inlet guide vane assembly for an industrial centrifugal compressor," in Energy Saving in the Design and Operation of Compressors, vol. 13, no. 1, pp. 11-22, Mechanical Engineering Publications Limited, 1996.

[11] M. Coppinger and E. Swain, "Performance prediction of an industrial centrifugal compressor inlet guide vane system," Proceedings of the Institution of Mechanical Engineers, Part A: Journal of Power and Energy, vol. 214, no. 2, pp. 153-164, 2000.

[12] A. Mohseni, E. Goldhahn, R. A. Van Den Braembussche, and J. R. Seume, "Novel IGV designs for centrifugal compressors and their interaction with the impeller," in Proceedings of the ASME Turbo Expo 2010: Power for Land, Sea, and Air, GT 2010, pp. 2019-2029, UK, June 2010.

[13] J. H. Mathews, Numerical Methods Using MATLAB Fourth Edition, Publishing House of Electronics Industry, Beijing, China, 2010.

[14] L. Piegl and W. Tiller, The NURBS Book, Springer Science \& Business Media, 2012.

[15] M. Imine and H. Nagahashi, "A new approach in polynomial and Bézier parametric piecewise modeling," Computer Networks, vol. 29, no. 14, pp. 1559-1570, 1997.

[16] J. Tang, J. Wang, and H. Liu, "A rapid prototyping parametric method of multi-blade centrifugal fan based on MATLAB," Blower \& Fan Technology, vol. 57, no. 2, pp. 42-47, 2015.

[17] L. Fang, B. Yang, and T. Gao, "Parameterization of Blade Based on Bezier-curve and Smooth Process of Leading and Trailing Edges," Compressor, Blower \& Fan Technology, no. 6, pp. 19-26, 2016.

[18] W. Zhang, X. Fang, and P. Wang, Interactive modeling program design of 3D turbine blade, Mechanical design and manufacturing,

[19] H. Zhang, X. Guo, and R. Dai, "Comparison of parametric parameterization methods in airship exterior design," Chinese Quarterly of Mechanics, vol. 32, no. 4, pp. 634-639, 2011.

[20] D. Zhang, Application Design of MATLAB Neural Network, China Machine Press, Beijing, China, 2012.

[21] L. Chen, Aerodynamic optimization design of compressor blade based on neural network and genetic algorithm [M.S. thesis], Northwestern Polytechnical University, Xi'an, China, 2007. 


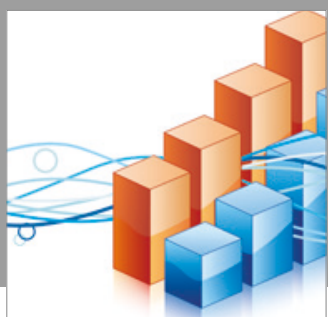

Advances in

Operations Research

vatersals

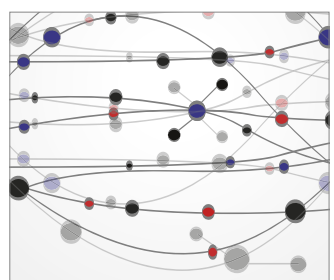

\section{The Scientific} World Journal
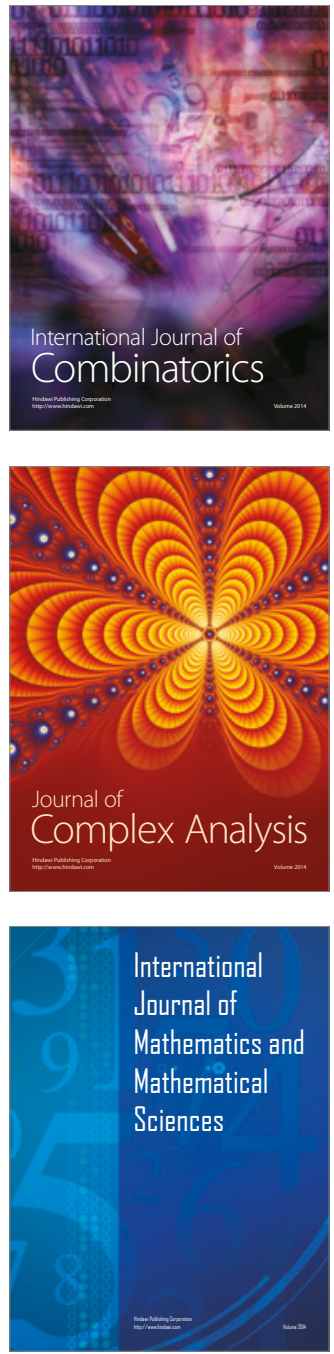
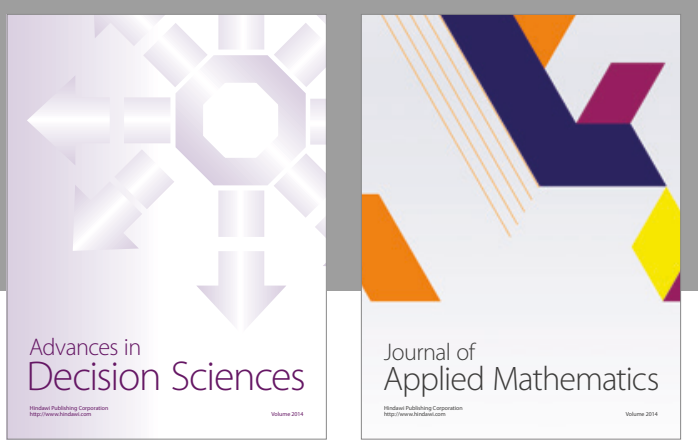

Algebra

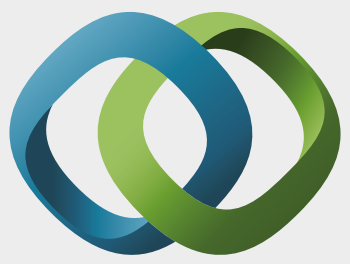

\section{Hindawi}

Submit your manuscripts at

https://www.hindawi.com
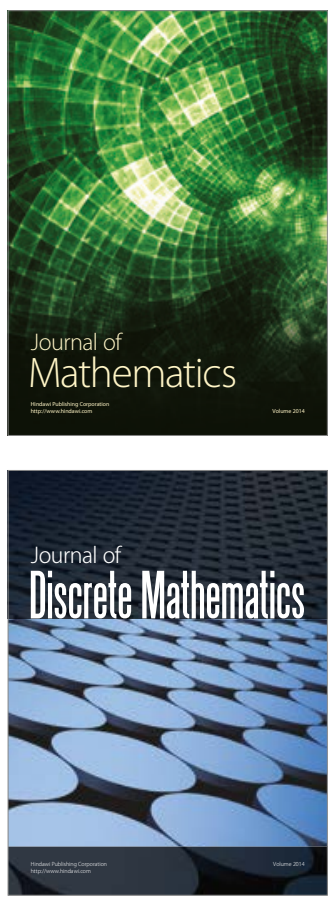

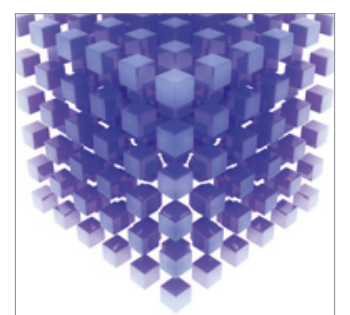

Mathematical Problems in Engineering
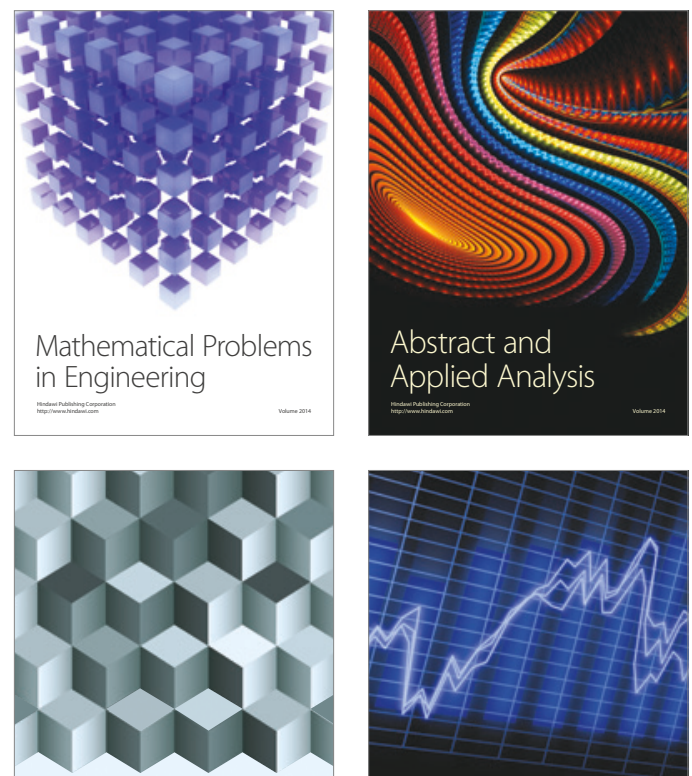

Journal of

Function Spaces

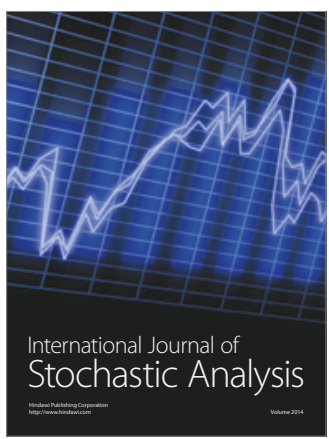

Probability and Statistics
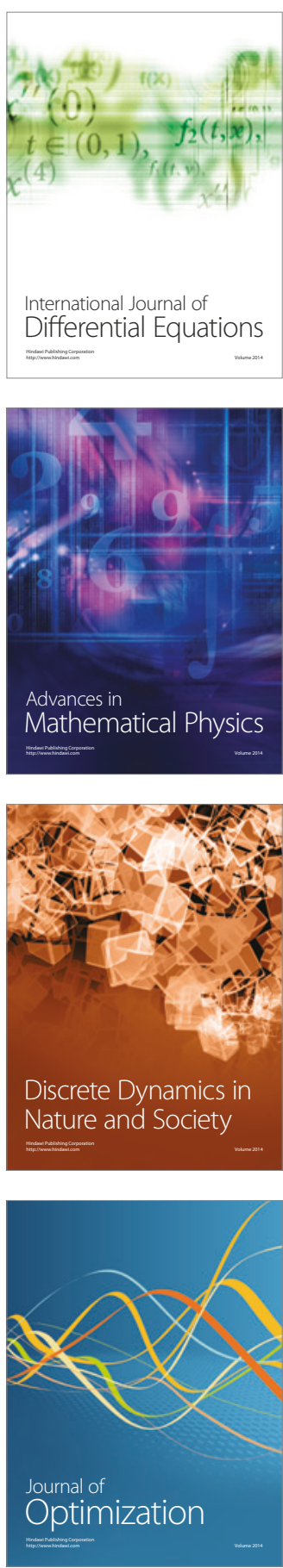